\title{
Altered regional homogeneity of prefrontal cortex in Parkinson's disease with mild cognitive impairment
}

\author{
De-Zhi Kang ${ }^{1 *}$, Fu-Xiang Chen ${ }^{1}$, Fu-Yong Chen ${ }^{1}$, Ying Liu, Gang Wu ${ }^{3}$, Liang-Hong Yu', Yuan-Xiang Lin ${ }^{1}$ \\ and Zhang-Ya Lin'
}

\begin{abstract}
Background: Mild cognitive impairment (MCl) is a common non-motor symptom of early Parkinson's disease (PD), but the neural mechanisms underlying it remain poorly understood. The aim of the present study was to investigate the characteristics of cognition-related brain activities in the PD patients with $\mathrm{MCl}$.

Methods: The brain fMRls and cognition tests were acquired in 39 PD patients and 22 healthy controls $(\mathrm{HC})$ from September 2013 to January 2015. The patients were divided into two groups: PD-MCI $(n=18)$ and PD with normal cognition (PDNC, $n=19$ ). we used resting state fMRI and a regional homogeneity (ReHo) method to explore patterns of intrinsic brain activity in patients with PD-MCl as compared with PDNC subjects and HC.

Results: Compared with the PDNC group, the PD-MCI group exhibited significantly increased ReHo in parts of the prefrontal cortex regions (e.g. right superior frontal gyrus, right middle frontal gyrus and orbitofrontal cortex). Compared to the HC group, a decrease of ReHo value in left thalamus was found in PD-MCl. However, this reduction was not found in the left thalamus of PDNC group, but in the above prefrontal regions $(p<0.05$, with Bonferroni correction).

Conclusions: These results demonstrate that the ReHo of prefrontal cortex in resting state is changed in PD patients with $\mathrm{MCl}$. The presence of $\mathrm{MCl}$ in PD may be attributed to abnormal regional activity in prefrontal cortex regions.
\end{abstract}

Keywords: Parkinson's disease, Mild cognitive impairment, Resting state fMRI, Regional homogeneity, Brain activity, Prefrontal cortex

\section{Background}

Mild cognitive impairment (MCI) is a common nonmotor feature of Parkinson's disease (PD). It affects multiple cognitive domains, such as executive function, memory, attention and visuospatial abilities $[1,2]$, and is associated with increasing age, duration of disease and disease severity [3]. The frequency of PD-MCI is estimated to be $42.5 \%$ in a large cohort of newly diagnosed PD participants [4], and with a higher risk progressed to dementia during follow-up [5], which may lead to poor quality of life [6]. Therefore, early identification of PD-MCI by its

\footnotetext{
* Correspondence: kdz99988@sina.com

${ }^{1}$ Department of Neurosurgery, The First Affiliated Hospital, Fujian Medical University, Fuzhou 350005, Fujian Province, China

Full list of author information is available at the end of the article
}

biomarkers have been one of the most popular research fields in recent years. However, the specific neural substrates underlying cognitive dysfunction in PD are still not well-known [7].

Resting state functional magnetic resonance imaging (rs-fMRI) is a cognitively unbiased imaging technique with high spatial and temporal resolution [8], and is widely thought to reflect spontaneous neural activity of the human brain relative to task-based [9], especially in the low-frequency band. Accumulating evidence has demonstrated that rs-fMRI is a effective and convenient method of mechanism detection, and monitoring the progression of PD [10]. Thus, there has been increasing interest in the use of rs-fMRI in PD and PD-related comorbidities [10-13]. 
Regional homogeneity ( $\mathrm{ReHo}$ ) is one of the important analysis methods developed on the basis of rs-fMRI, which measures the local synchronization of spontaneous neural activity by calculating the similarity between the time series of a given voxel and its nearest neighbors throughout the whole brain [14]. Unlike the functional connectivity, ReHo reflects the local features of brain activity in the resting state rather than the synchronization of activity between remote brain regions. To date, ReHo method has been extensively used to explore the alterations of functional activity in many neurological and psychiatric disorders, such as Alzheimer's disease [15], Schizophrenia [16, 17], depression [18, 19], and primary insomnia [20]. Likewise, this process has also been used to determine PD-related patterns of neural activity in the resting state $[10,11]$. For example, $\mathrm{Wu}$ and colleagues detected that PD patients showed significant $\mathrm{ReHo}$ alterations in extensive brain regions, including putamen, thalamus, cerebellum, and supplementary motor area, etc. Besides, they also found that the ReHo values in some of these regions were correlated with the disease severity measured by the Unified Parkinson's Disease Rating Scale (UPDRS) scores [10]. In another study, Zhang and colleagues revealed a distinct ReHo patterns in two different subtypes of PD, which primarily distributed over the striatal-thalamo-cortical and cerebello-thalamo-cortical loops [11]. To the best of our knowledge, rare rs-fMRI study focused on the effect of cognitive function decline on the patterns of neural activity in early PD patients. According to a recent study, PD-MCI patients displayed brain activity decreases in the cognitive corticostriatal loop, which included prefrontal cortex and caudate nucleus while planning a set shift task [12], and prefrontal hypometabolism was also found in metabolic studies on PD-MCI [21, 22]. Taken together, these neuroimaging studies indicate that there exists aberrant neural activity in PD-MCI patients, and ReHo changes are associated with different characteristics of the disease. Most importantly, there may be certain relationship between prefrontal cortex and cognitive dysfunction in PD-MCI patients.

We hypothesized that patients with PD-MCI would show ReHo alterations in cognition-related regions of the prefrontal cortex at the baseline state. With the purpose to investigate the cognition-related characteristics of ReHo, here, we used rs-fMRI and a ReHo measurement to assess regional intrinsic brain activity across the whole brain in healthy controls (HC), PD patients with $\mathrm{MCI}$, and those with normal cognitive function.

\section{Methods}

\section{Subjects}

Thirty-nine PD patients (30 males, 9 females, mean age of 61.9 years, age range 50-73 years) were recruited from September 2013 to January 2015 in the neurology clinic of the First Affiliated Hospital of Fujian Medical University. Patients were diagnosed by neurologists according to the UK Parkinson's Disease Society Brain Bank criteria [23]. Exclusion criteria included parkinsonism other than idiopathic PD; a history of brain surgery; special physical conditions not suitable for MRI scanning; diagnosed as PD dementia and other possible reasons that were responsible for cognitive function decline (such as head injury, stroke, epilepsy, metabolic abnormalities and major depression). HC group was comprised of 22 healthy volunteers matched for mean age, gender and level of education. The subjects were all right-handed and enrolled consecutively. The present study was approved by the local Ethics Committee of the First Affiliated Hospital of Fujian Medical University (Fujian, China), and written informed consent was obtained from all subjects.

\section{Clinical and neuropsychological assessment}

Neuropsychological tests were carried out with all subjects. Global cognition was assessed by trained neurologists by using Mini-Mental State Examination (MMSE) and Montreal Cognitive Assessment (MoCA). The MoCA scale has been validated as an effective assessment instrument for diagnosing PD-MCI [24], and with a score less than 26 was found to be the optimal cutoff point for diagnosis of cognitive deficits. The patients then were administered the UPDRS III and the Hoehn-Yahr stage to evaluate their motor severity and stage of the patient's parkinsonism while off their medications. All patients came in off all medications for at least $12 \mathrm{~h}$ for testing and subsequent imaging. PD-MCI was defined according to the Level I (abbreviated assessment) criteria at 1.5 Standard deviations below normative values, which was proposed by Movement Disorder Society Task Force in 2012 [24]. Patients who did not fulfill this criteria and had normal cognitive function were classified as PDNC group.

\section{Data acquisition}

All subjects were scanned on a 3.0 T SIEMENS MAGNETOM Verio MR imaging scanner (Siemens Medical Solutions, Erlangen, Germany) at the Department of Radiology using a standard head coil. Foam padding and earplugs were used to minimize head motion and noise. Rs-fMRI data were acquired using an echo-planar imaging (EPI) sequence with the following parameters: 31 slices, thickness $/ \mathrm{gap}=3.5 / 0.7 \mathrm{~mm}, \mathrm{TR}=2000 \mathrm{~ms}$, $\mathrm{TE}=30 \mathrm{~ms}$, flip angle $=90^{\circ}$, field of view $=200 \times$ $200 \mathrm{~mm}$, matrix $=64 \times 64$. For each subject, a total of 190 time points were obtained. During rs-fMRI acquisition, all subjects were asked to stay awake with their eyes closed, and to try not to think of anything in particular. In addition, before MRI data were acquisited, all individuals were scanned with $\mathrm{T} 2$-weighted images 
to exclude morphological abnormalities or intracranial lesions.

\section{Data preprocessing}

Functional data preprocessing was performed by the Data Processing Assistant for Resting-State fMRI (DPARSF, http://restfmri.net/forum/DPARSF) and the Resting State fMRI Data Analysis Toolkit software (REST, http:// www.restfmri.net/forum/REST), which were developed based on statistical parametric mapping (SPM8, http:// www.fil.ion.ucl.ac.uk/spm). The first 4 time points of fMRI data were discarded from analysis in consideration of magnetization stabilization and the adaption of subjects to the scanning environment, after which 186 time points remained. Subjects whose head motion parameters exceed more than $2 \mathrm{~mm}$ in any of the cardinal directions $(\mathrm{x}, \mathrm{y}, \mathrm{z})$ and $2^{\circ}$ of angular motion were excluded from further analysis. Preprocessing of the data contained several processes: (1) slice timing (reference slice is 31) was performed to correct the acquisition time delay between the different slices; (2) realignment to the first image, according to the estimated head movement, was performed to correct for geometrical displacements; (3) spatially normalized to the Montreal Neurological Institute (MNI) template by using T1 image unified segmentation, In this step, voxel size was resampled to $3 \mathrm{~mm}$ isotropic voxels; (4) Linear trends were removed and a temporal filter (0.01-0.08 Hz) was applied to reduce low-frequency drifts and physiological high-frequency respiratory and cardiac noise; Finally, the ReHo maps were spatially smoothed with a Gaussian filter of $4 \mathrm{~mm}$ of full width at half maximum (FWHM).

\section{Statistical analysis}

Group statistics were performed by using SPSS 20.0. Demographic and clinical characteristics differences among the three groups were compared by using oneway analysis of variance (ANOVA). For nonparametric data, Kruskal-Wallis test were used. The significance of group differences was set at $P<0.05$.

A one-way ANOVA was performed on the restingstate REST data to identify the differences between the PD-MCI, PDNC and $\mathrm{HC}$ groups. Voxels with a $\mathrm{p}$ value less than 0.05 and a cluster size greater than 228 voxels were considered significantly different (Alphasim corrected). Subsequently, the identified brain regions that showed significant differences were extracted as regions of interest (ROIs), and the ReHo values were conducted for a post hoc analysis. Multiple comparisons of the ReHo values between each pair of groups (PD-MCI vs PDNC, PD-MCI vs NC, PDNC vs NC) were performed using a two-sample two-tailed t-test at a threshold of $p<0.05$ (Bonferroni corrected).

\section{Results}

We acquired rs-fMRI data from 39 patients and 22 matched HC. No subject was excluded for none of them has large head motion. Among PD patients, eighteen of the patients fulfilled the new criteria for PD-MCI, and the rest of them were classified as PDNC [24]. Table 1 presents demographic and clinical features of each group. There were no significant differences in age, gender, duration of disease, education level and disease severity between the three groups $(p>0.05)$. As expected, patients with PD-MCI had significantly lower global cognition scores than the other two groups (both, $p<0.001$ ).

One-way ANOVA revealed that, among the three groups, ReHo has significant differences in the following regions: right superior frontal gyrus, right middle frontal gyrus, right orbitofrontal cortex, and left thalamus $(P<0.05$; AlphaSim corrected) (Fig. 1$)$. By identify the differences of ReHo values with a two-sample two-tailed t-test to each pair of groups, We found ReHo values were increased in the right superior frontal gyrus, right middle frontal gyrus and orbitofrontal cortex with no region decreased in the PD-MCI group comparing to

Table 1 Demographic and clinical features of subjects

\begin{tabular}{lllll}
\hline & $\mathrm{HC}(n=22)$ & PDNC $(n=21)$ & PD-MCl $(n=18)$ & $p$-value \\
\hline Gender (males/females) $^{a}$ & $16 / 6$ & $16 / 5$ & $14 / 4$ & 0.930 \\
Age (years) $^{\text {b }}$ & $61.95 \pm 5.27$ & $61.24 \pm 6.41$ & $62.83 \pm 5.38$ & 0.688 \\
Disease duration (years) $^{\mathrm{b}}$ & $\mathrm{NA}$ & $5.73 \pm 3.27$ & $6.62 \pm 4.55$ & 0.481 \\
Education (years) $^{\mathrm{a}}$ & $9.27 \pm 2.12$ & $10.38 \pm 3.49$ & $9.55 \pm 3.63$ & 0.435 \\
MoCA $^{\text {a }}$ & $27.86 \pm 1.39$ & $27.90 \pm 1.26$ & $20.72 \pm 2.42$ & $<0.001$ \\
MMSE $^{\text {a }}$ & $29.59 \pm 0.59$ & $29.38 \pm 0.80$ & $27.83 \pm 1.54$ & $<0.001$ \\
UPDRS III $^{\text {b }}$ & NA & $17.95 \pm 9.23$ & $24.00 \pm 10.98$ & 0.070 \\
H-Y Stage $^{\text {a }}$ & NA & $1.90 \pm 0.70$ & $2.44 \pm 0.98$ & 0.061 \\
\hline
\end{tabular}

Date are showed as means \pm standard deviations. PD Parkinson's disease, $H C$ healthy control, $P D N C$ PD with normal cognition, $P D-M C I$ PD with mild cognitive impairment, NA non available, MoCA Montreal Cognitive Assessment, MMSE Mini-Mental State Examination, UPDRS III Unified Parkinson's Disease Rating Scale part III, $H$-Y Stage Hoehn-Yahr stage

a $p$ values were calculated using Kruskal-Wallis test;

${ }^{\mathrm{b}} p$ values were calculated using one-way analysis of variance 


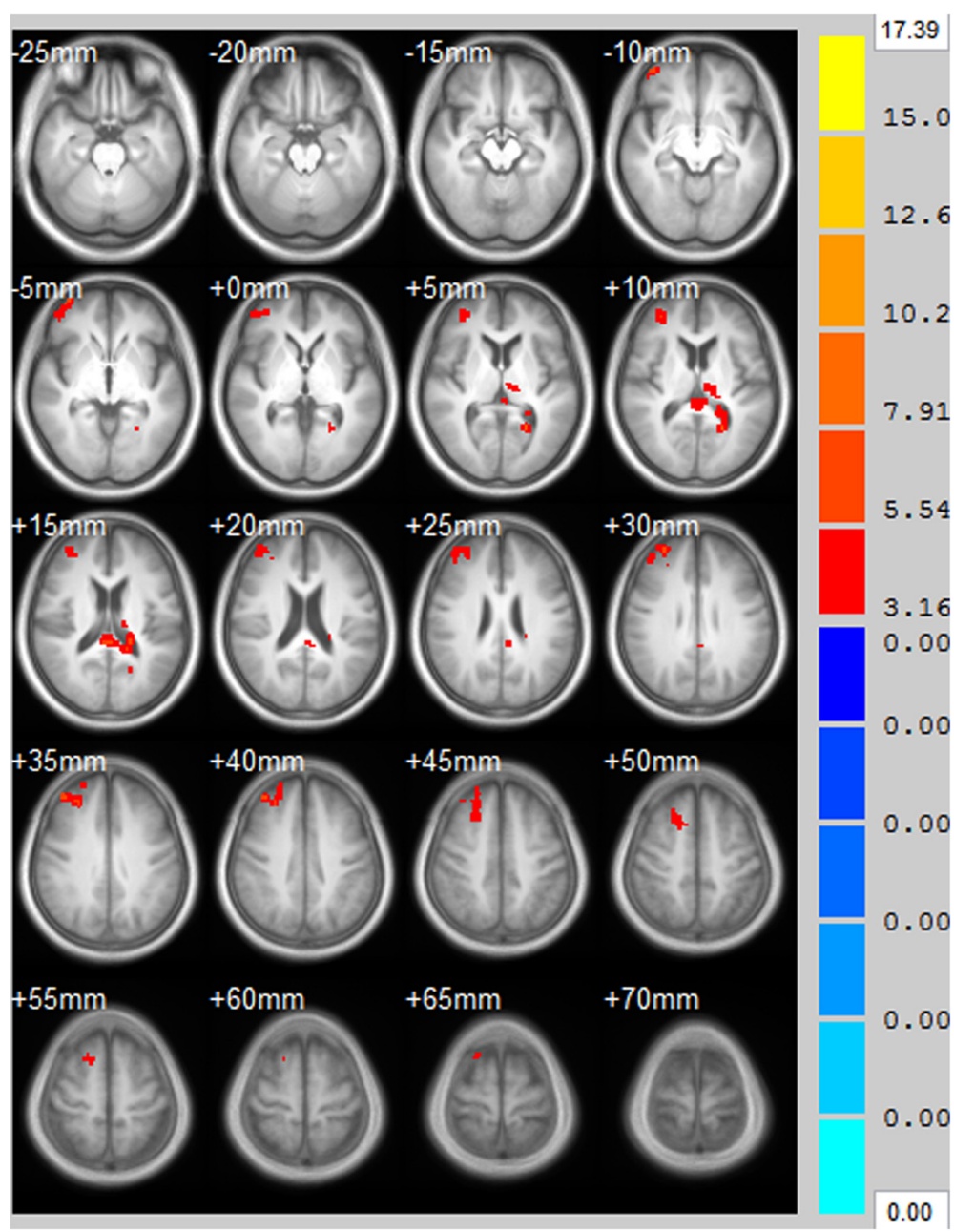

Fig. 1 Differences in ReHo values between the three groups of subjects. The threshold for displaying was set to $p<0.05$, alphasim corrected, cluster size $>228$. More details of these color regions are described in Table 2

the PDNC group, but decreased in the left thalamus that comparing to the $\mathrm{HC}$ group. Interestingly, compared with the $\mathrm{HC}$ group, the PDNC group also showed decreased ReHo values in the aforementioned regions other than left thalamus $(P<0.05$, Bonferroni corrected; Fig. 2$)$. In contrast, we did not find significant decline of ReHo values in any region in the $\mathrm{HC}$ group. More details of these regions are listed in Table 2.

\section{Discussion}

In this study, we utilized rs-fMRI and the ReHo assessment to investigate patterns of intrinsic brain activity in patients with PD-MCI as comparing to PD subjects with normal cognition and $\mathrm{HC}$. Our findings indicate that the ReHo values of regional brain activity were changed in PD patients in the resting state. Compared with the PDNC group, the PD-MCI group showed significant ReHo values increases in the right superior frontal gyrus, right middle frontal gyrus and orbitofrontal cortex
(BA8/9/10). These regions are the important elements of prefrontal cortex [25]. The results are accordance to our hypothesis that ReHo is altered in the cognition-related regions of the prefrontal cortex in patients with PD-MCI. Additionally, ReHo value was individually decreased in the left thalamus in patients with PD-MCI comparing to normal subjects.

Prefrontal cortex is not only a nub of associative circuit in the striatal-thalamo-cortical loops, but also an important part of the frontoparietal network [26]. The disruption of the above circuit or network, resulted from the functional abnormality of prefrontal cortex, has been linked to cognitive dysfunction in PD [27-30]. In the current study, We found increased ReHo values in the prefrontal cortex, including the right superior frontal gyrus, right middle frontal gyrus and orbitofrontal cortex of the PD-MCI patients. This is consistent with several imaging studies that have also revealed structural or functional abnormality in aforementioned regions in 

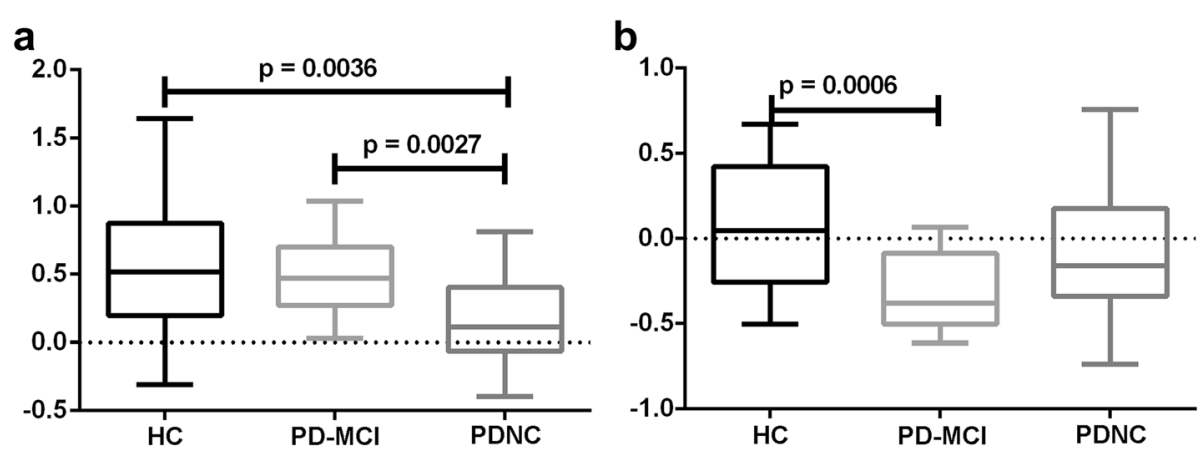

Fig. 2 Post hoc multiple comparisons of ReHo values in each region of interest (ROI). $X$ title represents three groups of subjects, $Y$ title indicates the ReHo values of each ROI. Significant results have been showed above the Box plot. $\mathbf{a}$, right superior frontal gyrus, right middle frontal gyrus and orbitofrontal cortex; $\mathbf{b}$, left thalamus

patients with PD-MCI compared with those with no cognitive impairment [12, 31-34]. Especially, in a recent fMRI study, Nagano-Saito et al. even proposed directly that the presence of MCI in PD affects brain activity in the prefrontal cortex and some other areas [12]. Considering these, we insist that there is important relevance between ReHo alternations in the prefrontal cortex and early cognition decline in PD patients. However, what causality between the altered ReHo in the prefrontal cortex and cognitive impairment in PD remains unclear in the current studies, and this needs to be clarified in the future.

The integrity of the thalamus is generally believed to be crucial for motor and cognitive function. We found ReHo decreased in left thalamus of the PD-MCI patients comparing to the normal subjects. ReHo declines in the local brain regions reflecting functional synchronization reductions in those areas may imply the existence of brain dysfunction [35]. To some extent, thalamic ReHo reduction in patients with $\mathrm{PD}-\mathrm{MCI}$ can be attributed to PD-related movement symptoms and cognitive deficits. Moreover, the ReHo alteration limited in left thalamus may be the result of compensatory mechanism of anti-parkinsonian medication, asymmetry of PD-onset or our cohort might be in a relatively early stage of cognitive decline.

In contrast to the $\mathrm{HC}$ group, the current study found that the PDNC patients showed ReHo attenuations in the right superior frontal gyrus, right middle frontal gyrus and orbitofrontal cortex. This is partially corresponded to previous researches [13, 36, 37], which have showed ReHo diminishment in the aforementioned regions of PDNC patients. However, in our study, we did not find significant increase of ReHo in the aforementioned regions in the PDNC group. This discrepancy may be due to the different subtypes of PD.

Meanwhile, we need to notice the limitations of our study. First, we only applied Level I criteria (abbreviated assessment) to define PD-MCI, which certainly provides less diagnostic certainty than level II and does not allow subsequent subtyping of PD-MCI. This constraint prevented us from further investigating the associations between alterations of ReHo and cognitive domains. However, the PD patients meet the level II criteria may be difficult to cooperate the tests. Second, the participants were predominantly males, but these patients were recruited consecutively and there were no gender difference among the three groups. Third, we are unable to observe the relevant alterations of ReHo caused to cognitive function decline because of this cross-sectional study, therefore, longitudinal follow-ups of this cohort and detailed neuropsychological tests should be conducted in future studies.

\section{Conclusions}

The current results in our study indicate that regional spontaneous neural activity in the resting state is altered in patients with PD-MCI, and these changes mainly located in prefrontal cortex regions, which may be related

Table 2 Brain regions of increased and decreased regional homogeneity among three groups

\begin{tabular}{|c|c|c|c|c|c|c|c|c|}
\hline \multirow[t]{2}{*}{ ROI } & \multirow[t]{2}{*}{ Regions } & \multirow[t]{2}{*}{ Hemisphere } & \multirow[t]{2}{*}{$\mathrm{BA}$} & \multirow{2}{*}{$\begin{array}{l}\text { Number } \\
\text { of voxels }\end{array}$} & \multirow{2}{*}{$\begin{array}{l}\text { Peak activation } \\
\text { strength (F) }\end{array}$} & \multicolumn{3}{|c|}{ Peak coordinates } \\
\hline & & & & & & $x$ & $y$ & z \\
\hline 1 & $\begin{array}{l}\text { Middle and superior frontal } \\
\text { gyrus, Orbitofrontal cortex }\end{array}$ & $\mathrm{R}$ & $8 / 9 / 10$ & 376 & 7.2721 & 30 & 33 & 36 \\
\hline 2 & Thalamus & L & / & 229 & 8.3344 & -21 & -57 & 9 \\
\hline
\end{tabular}

$R O /$ region of interest, $B A$ Brodmann area, The coordinates $\mathrm{x}, \mathrm{y}$ and $\mathrm{z}$ refer to the anatomical location, indicating standard stereotactic space as defined by Montreal Neurological Institute 
to the initial stages of cognitive function decline of PD. These findings add to the growing literature of abnormal intrinsic brain activity underlying cognitive deficits in early PD.

\section{Abbreviations}

PD: Parkinson's disease; HC: Healthy control; PDNC: PD with normal cognition; PD-MCl: PD with mild cognitive impairment; ReHo: Regional homogeneity; rs-fMRI: Resting state functional magnetic resonance imaging; UPDRS III: Unified Parkinson's Disease Rating Scale part III; MoCA: Montreal Cognitive Assessment; MMSE: Mini-Mental State Examination; H-Y Stage: Hoehn-Yahr stage; EPI: Echo-planar imaging; DPARSF: Data Processing Assistant for Resting-State fMRI; SPM: Statistical parametric mapping; MNI: Montreal Neurological Institute; REST: Resting State fMRI Data Analysis Toolkit software; FWHM: Full-width at half-maximum; ANOVA: Analysis of variance; ROl: Region of interest; BA: Brodmann area..

\section{Competing interests}

The authors declare that they have no competing interests.

\section{Authors' contributions}

D-ZK conceived and designed the experiments. F-XC drafted the manuscript. F-YC analyzed the data. YL performed the MRI scanning. GW and Z-YL helped to determine cognition scores, L-HY and Y-XL helped to collected patients and healthy controls. All authors read and approved the final manuscript.

\section{Acknowledgements}

We thank Center for Cognition and Brain Disorders, Hangzhou Normal University for data processing. The study was generously supported by the Fujian Province Natural Science Foundation (No. 2013 J01298), and also sponsored by key clinical specialty discipline construction program of Fujian, PRC.

\section{Author details}

'Department of Neurosurgery, The First Affiliated Hospital, Fujian Medical University, Fuzhou 350005, Fujian Province, China. ²Department of Radiology, The First Affiliated Hospital, Fujian Medical University, Fuzhou 350005Fujian Province, China. ${ }^{3}$ Department of Neurology, The First Affiliated Hospital, Fujian Medical University, Fuzhou 350005, Fujian Province, China.

\section{Received: 23 October 2015 Accepted: 23 January 2016}

\section{Published online: 18 March 2016}

\section{References}

1. Barone P, Aarsland D, Burn D, Emre M, Kulisevsky J, Weintraub D. Cognitive impairment in nondemented Parkinson's disease. Mov Disord. 2011:26(14):2483-95. doi:10.1002/mds.23919.

2. Aarsland D, Bronnick K, Williams-Gray C, Weintraub D, Marder K, Kulisevsky J, et al. Mild cognitive impairment in Parkinson disease: a multicenter pooled analysis. Neurology. 2010;75(12):1062-9. doi:10.1212/WNL. 0b013e3181f39d0e.

3. Litvan I, Aarsland D, Adler CH, Goldman JG, Kulisevsky J, Mollenhauer B, et al. MDS Task Force on mild cognitive impairment in Parkinson's disease: critical review of PD-MCI. Mov Disord. 2011;26(10):1814-24. doi:10.1002/mds.23823.

4. Yarnall AJ, Breen DP, Duncan GW, Khoo TK, Coleman SY, Firbank MJ, et al. Characterizing mild cognitive impairment in incident Parkinson disease: the ICICLE-PD study. Neurology. 2014;82(4):308-16. doi:10.1212/WNL. 0000000000000066

5. Pedersen KF, Larsen JP, Tysnes OB, Alves G. Prognosis of mild cognitive impairment in early Parkinson disease: the Norwegian ParkWest study. JAMA Neurol. 2013;70(5):580-6. doi:10.1001/jamaneurol.2013.2110.

6. Lawson RA, Yarnall AJ, Duncan GW, Khoo TK, Breen DP, Barker RA, et al. Quality of life and mild cognitive impairment in early Parkinson's disease: does subtype matter? J Park Dis. 2014;4(3):331-6. doi:10.3233/JPD-140390.

7. Ray NJ, Strafella AP. The neurobiology and neural circuitry of cognitive changes in Parkinson's disease revealed by functional neuroimaging. Mov Disord. 2012;27(12):1484-92. doi:10.1002/mds.25173.

8. Magnuson ME, Thompson GJ, Schwarb H, Pan WJ, McKinley A, Schumacher EH et al. Errors on interrupter tasks presented during spatial and verbal working memory performance are linearly linked to large-scale functional network connectivity in high temporal resolution resting state fMRI. Brain Imaging Behav. 2015. doi:10.1007/s11682-014-9347-3.

9. Wang K, Jiang T, Yu C, Tian L, Li J, Liu Y, et al. Spontaneous activity associated with primary visual cortex: a resting-state FMRI study. Cereb Cortex. 2008;18(3):697-704. doi:10.1093/cercor/bhm105.

10. Wu T, Long X, Zang Y, Wang L, Hallett M, Li K, et al. Regional homogeneity changes in patients with Parkinson's disease. Hum Brain Mapp. 2009;30(5):1502-10. doi:10.1002/hbm.20622.

11. Zhang J, Wei L, Hu X, Xie B, Zhang Y, Wu GR, et al. Akinetic-rigid and tremor-dominant Parkinson's disease patients show different patterns of intrinsic brain activity. Parkinsonism Relat Disord. 2015;21(1):23-30 doi:10.1016/j.parkreldis.2014.10.017.

12. Nagano-Saito A, Habak C, Mejia-Constain B, Degroot C, Monetta L, Jubault T, et al. Effect of mild cognitive impairment on the patterns of neural activity in early Parkinson's disease. Neurobiol Aging. 2014:35(1):223-31. doi:10.1016/j.neurobiolaging.2013.06.025.

13. Su M, Wang S, Fang W, Zhu Y, Li R, Sheng K, et al. Alterations in the limbic paralimbic cortices of Parkinson's disease patients with hyposmia under resting-state functional MRI by regional homogeneity and functional connectivity analysis. Parkinsonism Relat Disord. 2015;21(7):698-703. doi:10.1016/.parkreldis.2015.04.006

14. Zang Y, Jiang T, Lu Y, He Y, Tian L. Regional homogeneity approach to fMRI data analysis. Neuroimage. 2004;22(1):394-400. doi:10.1016/j.neuroimage. 2003.12.030

15. Wang $Y$, Zhao $X, X u$ S, Yu L, Wang L, Song $M$, et al. Using regional homogeneity to reveal altered spontaneous activity in patients with mild cognitive impairment. BioMed Res Int. 2015;2015:807093. doi:10.1155/2015/807093.

16. Gao B, Wang Y, Liu W, Chen Z, Zhou H, Yang J, et al. Spontaneous activity associated with delusions of schizophrenia in the left medial superior frontal gyrus: a resting-state fMRI study. PLoS One. 2015;10(7):e0133766. doi: 10.1371/journal.pone.0133766.

17. Chyzhyk D, Grana M, Ongur D, Shinn AK. Discrimination of schizophrenia auditory hallucinators by machine learning of resting-state functional MRI. Int J Neural Syst. 2015;25(3):1550007. doi:10.1142/S0129065715500070.

18. Huang $X$, Huang $P, L i ~ D$, Zhang Y, Wang T, Mu J, et al. Early brain changes associated with psychotherapy in major depressive disorder revealed by resting-state fMRI: evidence for the top-down regulation theory. Int J Psychophysiol. 2014;94(3):437-44. doi:10.1016/j.ijpsycho.2014.10.011.

19. Wu QZ, Li DM, Kuang WH, Zhang TJ, Lui S, Huang XQ, et al. Abnormal regional spontaneous neural activity in treatment-refractory depression revealed by resting-state fMRI. Hum Brain Mapp. 2011;32(8):1290-9. doi:10.1002/hbm.21108.

20. Wang T, Li S, Jiang G, Lin C, Li M, Ma X et al. Regional homogeneity changes in patients with primary insomnia. Eur Radiol. 2015. doi:10.1007/s00330-015-3960-4

21. Huang C, Mattis P, Perrine K, Brown N, Dhawan V, Eidelberg D. Metabolic abnormalities associated with mild cognitive impairment in Parkinson disease. Neurology. 2008;70(16 Pt 2):1470-7. doi:10.1212/01.wnl.0000304050. 05332.9c.

22. Lyoo CH, Jeong Y, Ryu YH, Rinne JO, Lee MS. Cerebral glucose metabolism of Parkinson's disease patients with mild cognitive impairment. Eur Neurol. 2010:64(2):65-73. doi:10.1159/000315036.

23. Hughes AJ, Daniel SE, Kilford L, Lees AJ. Accuracy of clinical diagnosis of idiopathic Parkinson's disease: a clinico-pathological study of 100 cases. J Neurol Neurosurg Psychiatry. 1992;55(3):181-4.

24. Litvan I, Goldman JG, Troster Al, Schmand BA, Weintraub D, Petersen RC, et al. Diagnostic criteria for mild cognitive impairment in Parkinson's disease: Movement Disorder Society Task Force guidelines. Mov Disord. 2012:27(3):349-56 doi:10.1002/mds.24893.

25. Ridderinkhof KR, van den Wildenberg WP, Segalowitz SJ, Carter CS. Neurocognitive mechanisms of cognitive control: the role of prefrontal cortex in action selection, response inhibition, performance monitoring, and rewardbased learning. Brain Cogn. 2004;56(2):129-40. doi:10.1016/j.bandc.2004.09.016.

26. Amboni M, Tessitore A, Esposito F, Santangelo G, Picillo M, Vitale C, et al. Resting-state functional connectivity associated with mild cognitive impairment in Parkinson's disease. J Neurol. 2015;262(2):425-34. doi:10.1007/s00415-014-7591-5.

27. Fellows LK, Farah MJ. The role of ventromedial prefrontal cortex in decision making: judgment under uncertainty or judgment per se? Cereb Cortex. 2007;17(11):2669-74. doi:10.1093/cercor/bhl176. 
28. Petrides $\mathrm{M}$. The role of the mid-dorsolateral prefrontal cortex in working memory. Exp Brain Res. 2000;133(1):44-54.

29. Japee S, Holiday K, Satyshur MD, Mukai I, Ungerleider LG. A role of right middle frontal gyrus in reorienting of attention: a case study. Front Syst Neurosci. 2015;9:23. doi:10.3389/fnsys.2015.00023.

30. Rinne JO, Portin R, Ruottinen $\mathrm{H}$, Nurmi E, Bergman J, Haaparanta M, et al. Cognitive impairment and the brain dopaminergic system in Parkinson disease: [18 F]fluorodopa positron emission tomographic study. Arch Neurol. 2000;57(4):470-5.

31. Melzer TR, Watts R, MacAskill MR, Pitcher TL, Livingston L, Keenan RJ, et al. Grey matter atrophy in cognitively impaired Parkinson's disease. J Neurol Neurosurg Psychiatry. 2012;83(2):188-94. doi:10.1136/jnnp-2011-300828.

32. Song SK, Lee JE, Park HJ, Sohn YH, Lee JD, Lee PH. The pattern of cortical atrophy in patients with Parkinson's disease according to cognitive status. Mov Disord. 2011:26(2):289-96. doi:10.1002/mds.23477.

33. Beyer MK, Janvin CC, Larsen JP, Aarsland D. A magnetic resonance imaging study of patients with Parkinson's disease with mild cognitive impairment and dementia using voxel-based morphometry. J Neurol Neurosurg Psychiatry. 2007;78(3):254-9. doi:10.1136/jnnp.2006.093849.

34. Ekman U, Eriksson J, Forsgren L, Mo SJ, Riklund K, Nyberg L. Functional brain activity and presynaptic dopamine uptake in patients with Parkinson's disease and mild cognitive impairment: a cross-sectional study. Lancet Neurol. 2012;11(8):679-87. doi:10.1016/S1474-4422(12)70138-2.

35. Sheng K, Fang W, Su M, Li R, Zou D, Han Y, et al. Altered spontaneous brain activity in patients with Parkinson's disease accompanied by depressive symptoms, as revealed by regional homogeneity and functional connectivity in the prefrontal-limbic system. PLoS One. 2014;9(1):e84705. doi:10.1371/journal.pone.0084705

36. Choe IH, Yeo S, Chung KC, Kim SH, Lim S. Decreased and increased cerebral regional homogeneity in early Parkinson's disease. Brain Res. 2013;1527:230-7. doi:10.1016/j.brainres.2013.06.027.

37. Yang H, Zhou XJ, Zhang MM, Zheng XN, Zhao YL, Wang J. Changes in spontaneous brain activity in early Parkinson's disease. Neurosci Lett. 2013:549:24-8. doi:10.1016/..neulet.2013.05.080

\section{Submit your next manuscript to BioMed Central and we will help you at every step:}

- We accept pre-submission inquiries

- Our selector tool helps you to find the most relevant journal

- We provide round the clock customer support

- Convenient online submission

- Thorough peer review

- Inclusion in PubMed and all major indexing services

- Maximum visibility for your research

Submit your manuscript at www.biomedcentral.com/submit

) Biomed Central 